\title{
Diagnóstico post mortem de Fasciola hepatica en bovinos faenados en la planta de beneficio de Sogamoso (Boyacá, Colombia)
}

\author{
Post mortem diagnosis of Fasciola hepatica in cattle slaughtered at the \\ Sogamoso, Boyacá processing plant (Boyacá, Colombia) \\ Melissa Camila Ortiz-Pineda ${ }^{1}$, Omar Alexander Archila-Barrera ${ }^{1}$, \\ Diana María Bulla-Castañeda ${ }^{1,3}$, Adriana María Díaz-Anaya ${ }^{1}$, \\ Julio Cesar Giraldo Forero' ${ }^{2}$, Diego José Garcia-Corredor', \\ Martín Orlando Pulido-Medellín ${ }^{1}$
}

\section{Resumen}

El objetivo del estudio fue determinar la prevalencia de distomatosis hepática en bovinos faenados en la planta de beneficio de Sogamoso (Boyacá, Colombia), y definir la técnica diagnóstica más efectiva para establecer la presencia del parásito. Se estableció un tamaño muestral de 343 individuos teniendo en cuenta la tasa de sacrificio mensual de la planta. Se tomaron muestras de heces, sangre y contenido biliar y se colectaron los parásitos adultos del ducto biliar. En el examen coproparasitológico se utilizó la técnica de Ritchie modificada, el contenido biliar fue analizado mediante sedimentación y para el diagnóstico serológico se implementó un ELISA in house estandarizado a partir de parásitos adultos de Fasciola hepatica. El 29.7\% (102/343) de los individuos muestreados

\footnotetext{
${ }^{1}$ Grupo de Investigación en Medicina Veterinaria y Zootecnia - GIDIMEVETZ, Universidad Pedagógica y Tecnológica de Colombia, Tunja, Colombia

${ }^{2}$ Grupo de Investigación en Parasitología y Microbiología Tropical-GIPAMT, Programa de Biología, Facultad de Ingenierías, Administración y Ciencias Básicas, Universidad INCCA de Colombia, Bogotá, Colombia

${ }^{3}$ E-mail: diana.bulla@uptc.edu.co
}

Recibido: 25 de enero de 2021

Aceptado para publicación: 31 de julio de 2021

Publicado: 27 de octubre de 2021

CLos autores. Este artículo es publicado por la Rev Inv Vet Perú de la Facultad de Medicina Veterinaria, Universidad Nacional Mayor de San Marcos. Este es un artículo de acceso abierto, distribuido bajo los términos de la licencia Creative Commons Atribución 4.0 Internacional (CC BY 4.0) [https:// creativecommons.org/licenses/by/4.0/deed.es] que permite el uso, distribución y reproducción en cualquier medio, siempre que la obra original sea debidamente citada de su fuente original 
fueron positivos a la presencia de $F$. hepatica en por lo menos una de las técnicas empleadas. La evaluación post mortem presentó el valor más alto $(19.8 \% ; 68 / 343)$, seguido por el ELISA in house (13.7\%; 47/343), detección de huevos en bilis $(117 \%$; 40/343) y la técnica coprológica $(7.3 \% ; 25 / 343)$.

Palabras clave: Fasciola hepatica, bovinos, prevalencia, ELISA

\section{AbSTRaCT}

The aim of this study was to determine the prevalence of hepatic dystomatosis in cattle slaughtered in the Sogamoso slaughterhouse (Boyacá, Colombia), and to define the most effective diagnostic technique to establish the presence of the parasite. A sample size of 343 individuals was established considering the monthly slaughter rate of the plant. Stool, blood and bile samples were taken, and adult parasites were collected from the bile duct. The modified Ritchie technique was used in the coproparasitological examination, the bile content was analyzed by sedimentation and a standardized inhouse ELISA was implemented for serological diagnosis from adult Fasciola hepatica parasites. The results showed that $29.7 \%$ (102/343) of the individuals sampled were positive for the presence of $F$. hepatica in at least one of the techniques used. The post mortem evaluation presented the highest value $(19.8 \% ; 68 / 343)$, followed by the inhouse ELISA $(13.7 \% ; 47 / 343)$, detection of eggs in bile $(117 \% ; 40 / 343)$ and the coprological technique $(7.3 \% ; 25 / 343)$.

Key words: Fasciola hepatica, cattle, prevalence, ELISA

\section{INTRODUCCIÓN}

La distomatosis hepática o fascioliasis es una enfermedad zoonótica de origen parasitario y distribución mundial (López et al., 2017), que afecta mayormente a los rumiantes y ocasionalmente al ser humano (Valderrama Pomé, 2016). Su agente etiológico es el trematodo Fasciola hepatica, un parásito del filo Platyhelminthes, clase Trematoda, orden Echinostomida y familia Fascioloidae (ITIS, 2019).

La fascioliasis es considerada como una de las enfermedades con mayor propagación a nivel mundial (Mas Coma et al., 2009), siendo causante de severas pérdidas económicas, las cuales han sido valoradas por encima de dos millones de dólares al año (Mehmood et al., 2017), mientras que en Colombia se estiman pérdidas anuales de
\$1.927 millones de pesos (US\$ 530 815) (Ortega et al., 2017), debido mayormente a la mortalidad de los animales, el decomiso de los hígados en las plantas de sacrificio, y por reducción en la ganancia de peso, producción de leche y rendimiento reproductivo (Rojo-Vásquez et al., 2012).

La fascioliasis humana tiene una gran importancia en salud pública, debido al aumento de su incidencia en la cordillera andina, cuyos países representan las principales zonas endémicas (Mas-Coma et al., 2005). La distribución geográfica de $F$. hepatica es amplia, encontrándose principalmente en Europa, América y Oceanía (Marcilla et al., 2002; Pereira et al, 2016). Se reportó una prevalencia de $78.8,75.8$ y $86.9 \%$ para Inglaterra, Escocia y Gales en vacas lecheras (Howell et al., 2015), de 27.4\% en Egipto (El Damaty et al., 2018), de $42.4 \%$ en Irán (Zaraei et al., 2019) y de 60\% en Etiopía 
(Asfaw, 2019), en tanto que para el continente americano se han reportado prevalencias entre 18.3 y $86.8 \%$ en ovinos y bovinos (Palacio et al., 2017; Quevedo et al., 2018; Jara et al., 2018). De igual forma en Colombia, se reconocen áreas endémicas de fascioliasis como los departamentos de Boyacá, Nariño, Antioquia y Cundinamarca (Becerra, 2001). Por consiguiente, se han definido prevalencias de $5.9 \%$ (Blanco et al., 1996), 42.2\% (García Murillo et al., 2009), 74.7\% (Castro y Becerra, 2011), 13.1\% (Bedoya et al., 2012), 3.7\% (Recalde-Reyes et al., 2014), 7.8\% (Pulido et al., 2014) y $22.8 \%$ (Correa et al., 2016).

El método de diagnóstico común para la fasciolosis en rumiantes es mediante la detección de huevos de $F$. hepatica en el examen coprológico, principalmente por medio de la técnica de Dennis (Sierra et al., 2017). Sin embargo, la frecuencia de falsos positivos en estas pruebas puede ser elevada debido al periodo de prepatencia y a la ovoposición irregular (Kajugu et al., 2015), así como de falsos positivos debido a la presencia de huevos que permanecen en el ducto biliar luego de las desparasitaciones (Sargison, 2012). De otra parte, se disponen de otros métodos de diagnóstico que pueden permitir la identificación de parásitos adultos en los canalículos hepáticos y de huevos en líquido biliar (Giraldo Forero et al., 2016), los cuales pueden considerarse como diagnósticos tardíos, pero útiles para el control sanitario.

Las pruebas de inmunodiagnóstico tienen una mayor sensibilidad que las pruebas coprológicas, pues permiten revelar la presencia del parásito en los canales hepáticos luego del periodo prepatente (Kajugu et al., 2015). Ante esto, el propósito del estudio fue determinar la prevalencia de distomatosis hepática en bovinos faenados en la planta de beneficio de Sogamoso (Boyacá), así como identificar la técnica diagnóstica más efectiva para establecer la presencia del parásito.

\section{Materiales y MéTodos}

\section{Lugar de Muestreo}

El municipio de Sogamoso está localizado en la región centro-oriente del departamento de Boyacá (Colombia). Se encuentra a una altitud de $2569 \mathrm{~m}$ y presenta una temperatura promedio anual de $18{ }^{\circ} \mathrm{C}$ (Alcaldía Municipal de Sogamoso, 2018).

\section{Tamaño de la Muestra}

Se trabajó con bovinos faenados provenientes de la planta de beneficio de Sogamoso, caracterizada por su alto volumen de sacrificio mensual procedente de municipios de los departamentos de Boyacá y Casanare. Se estableció un tamaño muestral de 343 individuos Holstein, Normando y cruzados mayores de 1 año, mediante el programa estadístico Open Epi, teniendo en cuenta una tasa de sacrificio mensual de la planta de 3141 bovinos. Se registraron los datos de los animales en fichas epidemiológicas durante el momento de sacrificio, tales como edad, sexo, raza y origen. Esta información fue proporcionada por las guías de movilización animal emitidas por el Instituto Colombiano Agropecuario (ICA).

\section{Colecta y Procesamiento de Muestras}

Las muestras de materia fecal fueron recolectadas directamente del recto y dispuestas en recipientes plásticos y almacenadas en cavas refrigeradas a $4{ }^{\circ} \mathrm{C}$. Además, se tomaron muestras de sangre $(10 \mathrm{ml})$ en tubos tapa roja por punción yugular. Luego del sacrificio y durante el eviscerado se recolectó el contenido biliar en frascos de plástico y se almacenaron en cavas de icopor a $4{ }^{\circ} \mathrm{C}$. La inspección directa de los hígados permitió la recolección de parásitos alojados en el ducto biliar (García Murillo et al., 2009). Los parásitos fueron preservados en alcohol al 70\% (Kooshan et al., 2010). 
Las muestras fueron trasladadas al Laboratorio de Parasitología Veterinaria de la Universidad Pedagógica y Tecnológica de Colombia (UPTC). Para la evaluación coproparasitológica se emplearon $4 \mathrm{~g}$ de materia fecal y se utilizó la técnica de Ritchie modificada para la identificación de huevos, ooquistes y larvas (Botero y Restrepo, 2005). Las muestras de sangre fueron centrifugadas a $2438 \mathrm{~g}$ durante 10 minutos para la obtención del suero (Figueiredo et al., 2017). El contenido biliar fue centrifugado a $3483 \mathrm{~g}$ durante 10 minutos y los precipitados se utilizaron para el reconocimiento de los estadios ovales de $F$. hepatica mediante microscopía directa (Giraldo Forero et al., 2016). Para el diagnóstico serológico se implementó un ELISA in house (Se 96\%, Sp 98\%, VP+ 96\% y VP- 98\%) estandarizado a partir de parásitos adultos de $F$. hepatica colectados de bovinos faenados en Boyacá, los cuales fueron lavados con solución salina y conservados en Buffer Fosfato de Sodio estéril (PBS, $\mathrm{pH}$ 7.2) suplementado con antibiótico (Giraldo Forero et al., 2016).

Para la elaboración del extracto antigénico, cada ejemplar de F. hepatica se homogenizó en una solución de PBS estéril (pH 7.2) y se llevó a delipidado con éter anhidro. Cada tratamiento se agitó durante 24 horas a $4{ }^{\circ} \mathrm{C}$. Finalmente, las muestras se sonicaron con ultrasonido, seguido de ultra centrifugación de la que se conservó el sobrenadante. Se realizó cuantificación proteica por medio de la técnica de Bradford con diluciones seriadas de albumina sérica bovina (ASB) para conocer la concentración del extracto antigénico. Luego se obtuvo un perfil electroforético en gel de AcrilamidaBisacrilamida. Para el proceso de extracción y purificación de las proteínas se separaron los componentes proteicos en geles de electroforesis de SDS-PEAGE al 10\%. Las fracciones antigénicas de interés se obtuvieron por procesos de elución y el corte de los geles producto de perfiles electroforéticos preparativos con fracciones antigénicas de
14 y $19 \mathrm{kDa}$. Se evaluaron nuevos perfiles electroforéticos para la caracterización de los extractos antigénicos totales en diferentes concentraciones. Las fracciones más antigénicas, dieron lugar a la optimización de la prueba.

Como control positivo se seleccionaron sueros de animales en los que mediante examen post mortem se identificó la presencia del parásito adulto en ducto biliar o de huevos en contenido biliar y que al realizar la titulación en bloque en la prueba de serodiagnóstico seleccionada, se identificaron títulos de anticuerpos valorados por espectrofotometría (tipo IgG) y que además superaran el punto de corte calculado para la prueba. Los sueros control negativo fueron seleccionados de animales que no cumplieron con los criterios establecidos para los sueros control positivo.

El punto de corte para la prueba de Elisa in house se calculó mediante la sumatoria de los valores de absorbancia, y a su promedio aritmético se le sumo dos desviaciones estándar. El punto de corte para la detección de anticuerpos IgG anti $F$. hepatica fue de 0.300 .

\section{Análisis Estadístico}

El estudio realizado fue de tipo descriptivo, de corte transversal con muestreo aleatorio simple. El procesamiento de la información se llevó a cabo mediante el software estadístico IBM SPSS Statistics 19 utilizando las pruebas Q de Cochran y McNemar.

\section{Consideraciones éticas}

El estudio se realizó bajo las condiciones de la Ley 576 del 2000 y la Ley 84 de 1989 de la República de Colombia. Se obtuvo consentimiento informado por parte de los propietarios de los bovinos antes de la recolección de las muestras. 
Cuadro 1. Prevalencia de Fasciola hepatica en bovinos faenados en Boyacá, Colombia de acuerdo con la técnica diagnóstica y el sexo de los animales muestreados

\begin{tabular}{cccccc}
\hline \multirow{2}{*}{ Técnica } & Sexo & \multicolumn{3}{c}{ Muestras } & Prevalencia \\
\cline { 3 - 4 } & & $\mathrm{n}$ & Positivos & \multirow{2}{*}{ P) } & P-valor \\
\hline Visualización post- & $\mathrm{H}$ & 148 & 25 & 16.9 & \multirow{2}{*}{0.235} \\
mortem & $\mathrm{M}$ & 195 & 43 & 22.1 & \\
ELISA in house & $\mathrm{H}$ & 148 & 17 & 11.5 & \multirow{2}{*}{0.298} \\
Bilis (Microscopía & $\mathrm{M}$ & 195 & 30 & 15.4 & \\
directa) & $\mathrm{H}$ & 148 & 16 & 10.8 & \multirow{2}{*}{0.669} \\
Coprología & $\mathrm{M}$ & 195 & 24 & 12.3 & \\
(Ritchie) & $\mathrm{H}$ & 148 & 9 & 6.1 & \multirow{2}{*}{0.454} \\
\hline
\end{tabular}

\section{Resultados}

La presencia de $F$. hepatica en los bovinos del estudio fue mayor al realizar la evaluación post mortem (19.8\%; 68/343), seguido por la técnica de ELISA in house $(13.7 \%$, $47 / 343)$, detección de huevos en bilis ( $11.7 \%$; $40 / 343)$ y la técnica coprológica $(7.3 \% ; 25 /$ 343). Por otra parte, si bien se observó una mayor prevalencia en machos que en hembras, las diferencias no fueron significativas (Cuadro 1).

La prueba Q de Cochran determinó una asociación estadística significativa entre las técnicas implementadas. Por otra parte, la prueba de McNemar evidenció diferencias significativas entre el número de casos positivos entre el análisis coprológico y el post mortem $(\mathrm{p}=0.000)$, análisis coprológico y ELISA in house $(\mathrm{p}=0.011)$, análisis biliar y post mortem $(\mathrm{p}=0.000)$ y post mortem y ELISA in house $(\mathrm{p}=0.001$, mientras que no hubo diferencias significativas entre el análisis coprológico y el análisis biliar $(\mathrm{p}=0.64)$ y el análisis biliar el el ELISA in house $(\mathrm{p}=$ 0.324) (Cuadro 2).

\section{Discusión}

Se han reportado estudios de prevalencia de distomatosis hepática en varios departamentos de Colombia, donde los valores de las prevalencias encontradas estuvieron influenciados por el método diagnóstico. Así, Giraldo Forero et al. (2016) reportaron una prevalencia de $32.4 \%$ al detectar los parásitos en los conductos biliares, en tanto que determinando coproantígenos en heces de bovinos faenados en Antioquia se reportaron valores de 5.9\% (Blanco et al., 1996) y de 13.1\% (Bedoya et al., 2012). Estas diferencias no solo pueden deberse a la técnica empleada, sino además, al número de animales muestreados.

La variabilidad de las prevalencias encontradas puede deberse, asimismo, al potencial del trematodo durante la etapa de desarrollo, así como al huésped y su respuesta inmune (De Ayala et al., 2014). Sumado a esto, intervienen factores como la edad del huésped (Soca Pérez et al., 2016), el contenido de agua en la materia fecal (Iturbe Espinoza y Muñiz Pareja, 2011), la cantidad 
Cuadro 2. Asociación entre técnicas implementadas para el diagnóstico de $F$. hepatica en bovinos faenados en la planta de beneficio de Sogamoso, Boyacá

\begin{tabular}{lcccccccc}
\hline Casos & $\begin{array}{c}\text { Copro- } \\
\text { logía }\end{array}$ & $\begin{array}{c}\text { Post } \\
\text { mortem }\end{array}$ & $\begin{array}{c}\text { Copro- } \\
\text { logía }\end{array}$ & $\begin{array}{c}\text { ELISA } \\
\text { in house }\end{array}$ & Bilis ${ }^{1}$ & $\begin{array}{c}\text { Post } \\
\text { mortem }\end{array}$ & $\begin{array}{c}\text { Post } \\
\text { mortem }\end{array}$ & $\begin{array}{c}\text { ELISA } \\
\text { in house }\end{array}$ \\
\hline $\begin{array}{l}\text { Positivos } \\
\text { Positivos } \\
\text { en común }\end{array}$ & 25 & 68 & 25 & 47 & 40 & 68 & 68 & 47 \\
\hline P-valor & 4 & & \multicolumn{2}{c}{2} & & 26 & & 38 \\
\hline
\end{tabular}

${ }^{1}$ Microscopía directa

de muestra y el número de alícuotas analizadas por muestra (Uribe et al., 2012), todos los cuales contribuyen a presentar variaciones en la detección de los huevos de $F$. hepatica.

La técnica diagnóstica ELISA in house fue la herramienta que presentó el segundo resultado más alto (13.7\%). En Colombia, la implementación de la prueba de ELISA ha reportado prevalencias de $40 \%$ en Boyacá (García Murillo et al., 2009) y de 5.9\% detectando coproantígenos del trematodo (Blanco et al., 1996) y de $80 \%$ de positividad en muestras de leche mediante un ELISA indirecto en Antioquia (Uruburu et al., 2013). Resultados diferentes fueron reportados en el Reino Unido $(79.7 \%$ en muestras de leche) por Howell et al. (2015), de 16-58\% en Bolivia por Hillyer et al. (1996) y de $23.1 \%$ en Perú por Valencia et al. (2005).

La herramienta diagnóstica que reportó la prevalencia más alta en los bovinos faenados fue el examen post mortem (19.8\%); sin embargo, es una técnica de diagnóstico tardío para la detección de la fascioliasis (Espino et al., 2000). En Colombia se reportan prevalencias de $24.4 \%$ en Boyacá (García Murillo et al., 2009) y de $32.4 \%$ en Cundinamarca (Giraldo Forero et al., 2016) mediante esta técnica, en tanto que en América Latina se reportan valores entre
28.2 y 35.5\% (Chagas et al., 2011; Brito et al., 2012; Palacio et al., 2017). Los resultados del presente estudio no concuerdan con los obtenidos por García Murillo et al. (2009), quienes encuentran más casos positivos con la técnica de ELISA que con la identificación de los parásitos en el hígado.

La ausencia de asociación entre el sexo de los animales con la presentación del parásito coincide con otras investigaciones (García Murillo et al., 2009; Afshan et al., 2013; Giraldo Forero et al., 2016), aunque se encontró que los machos presentaron prevalencias numéricas más altas respecto a las hembras en las cuatro herramientas diagnósticas.

Se presentaron diferencias estadísticas significativas entre las cuatro técnicas diagnósticas. Asimismo, se estableció que la concordancia se presentó entre las herramientas que estaban asociadas con el diagnóstico post mortem, lo cual podría deberse a que está técnica es la más efectiva para la detección del parásito en los rumiantes. De otra parte, Blanco et al. (1996) establecieron una relación del $100 \%$ entre los casos positivos a ELISA (11/183) y a la inspección directa de hígados (11/183). No obstante, la presencia de la duela adulta en el hígado no garantiza la aparición de huevos en el contenido biliar o en heces (Chirinos et al., 2000; Martínez et al., 2013). 


\section{ConClusiones}

- Se encontraron diferencias significativas entre las cuatro técnicas diagnósticas para Fasciola hepatica, especialmente con la visualización del trematodo en el hígado.

- No se encontró relación estadística entre el sexo de los bovinos y la presentación del parásito.

- Se pudo determinar que la presencia de los trematodos adultos en el hígado no está completamente relacionada con la detección de huevos y la presencia de seropositividad.

\section{Literatura Citada}

1. Afshan K, Qayyum M, Rizvi SSR, Mukhtar M, Mushtaq M, Miller JE. 2013. Serological and coprological comparison for rapid diagnosis of Fasciola hepatica infection in small ruminants from sub-tropical area of Pakistan. Small Ruminant Res 113): 267272. doi: 10.1016/j.smallrumres.2013.01.020

2. Alcaldía Municipal de Sogamoso. 2018. Alcaldía Municipal de Sogamoso, Boyacá. [Internet]. Disponible en: http:/ /www.sogamoso-boyaca.gov.co/

3. Asfaw A. 2019. Prevalence and economic significance of bovine fasciolosis in Bale Rural Abattoir, Ethiopia. Acad Agri J 3: 541-550.

4. Becerra Rozo WM. 2001. Consideraciones sobre estrategias sostenibles para el control de Fasciola hepatica en Latinoamérica. Rev Colomb Cienc Pec 14: 28-35.

5. Bedoya AJ, Hurtado Y, Pérez, J, Solano S, Úsuga MV, Vanegas M, Gómez $C$, et al. 2012. Primer registro de focos de fasciolosis y paramfistomosis en bovinos doble propósito, Gómez Plata, Antioquia, Colombia. Hechos Microbiol 3: 31-39.
6. Blanco A, Jaramillo G, Restrepo, JG 1996. Detección de coproantígenos de Fasciola hepatica por ELISA en bovinos sacrificados en el Matadero Municipal de Medellín. Rev Colomb Cienc Pec 9: 46-48.

7. Botero D, Restrepo M. 2005. Conceptos generales sobre parasitología. En: Parasitosis humanas. $4^{\circ}$ ed. Medellin, Colombia: Corporación para Investigaciones Biológicas. p 409-436.

8. Brito E, Hernández MA, Fe, PDS, Silveira EA. 2012. Prevalencia, decomisos de hígado y pérdidas económicas por Fasciola hepatica en mataderos bovinos de tres provincias de la región central de Cuba. REDVET 9(4). [Internet]. Disponible en: https://www.redalyc.org/ pdf/636/63613155004.pdf

9. Castro N, Becerra W. 2011. Foco de fasciolosis ovina en hacienda en la vereda Presidente, municipio de Chitagá, Norte de Santander, Colombia. Bistua 9: 64-72.

10. Chagas C, Batista Carneiro M, Rauta de Avelar B, Molinari Donatele D, Vilhena Freire I, Salim Pereira M. 2011. Prevalence of liver condemnation due to bovine fasciolosis in Southern Espírito Santo: temporal distribution and economic losses. Rev Bras Parasitol V 20: 49-53. doi: 10.1590/s1984-29612011000100010

11. Chirinos A, de Chirinos $N$, Roman $R$, HómezG, Pirela H, Rodríguez $N$. 2000. Distomatosis hepática bovina a nivel de dos mataderos industriales del estado Zulia, Venezuela. Rev Cient-Fac Cien V 10: 297-302.

12. Correa S, Martínez YL, López JL, Velásquez LE. 2016. Evaluación de la técnica modificada de Dennis para el diagnóstico de fasciolosis bovina. Biomédica 36: 64-68. doi: 10.7705/ biomedica.v36i2.2875

13. De Ayala Fernández JA, Cebrián, EM, Lazcano I, González F. 2014. Infecciones por trematodos: esquistosomiasis, fascioliasis, opistorquiasis, 
clonorquiasis y paragonimiasis. Medicine 11: 3115-3128. doi: 10.1016/S03045412(14)70748-6

14. El Damaty HM, Mahmmod YS, Gouda SM, Sobhy NM. 2018. Epidemiological and ultrasonographic investigation of bovine fascioliasis in smallholder production system in Eastern Nile Delta of Egypt. Prev Vet Med 158: 35-42. doi: 10.1016/j.prevetmed.2018.07.009

15. Espino AM, Borges A, Duménigo BE. 2000. Coproantigenos de Fasciola hepatica de posible utilidad en el diagnóstico de la fascioliasis. Rev Panam Salud Publ 7: 225-231. doi: 10.1590/ S1020-49892000000400003

16. Figueiredo Marques G, Augusto Pompei JC, Martini M. 2017. Manual veterinario de toma y envío de muestras 2017. Cooperación Técnica MAPA/ OPS/PANAFTOSA para el Fortalecimiento de los Programas de Salud Animal de Brasil. Rio de Janeiro: PANAFTOSA - OPS/OMS. 218 p.

17. García-Murillo ME, Granados-Hurtado SJ, Pulido-Medellín MO, Andrade-Becerra RJ. 2009. Comparación de métodos diagnósticos para Fasciola hepatica en el matadero de Chiquinquirá (Boyacá). Cien Agric 7: 71-79.

18. Giraldo Forero JC, Díaz Anaya AM, Pulido Medellín MO. 2016. Prevalencia de Fasciola hepatica en bovinos sacrificados en la planta de beneficio del municipio de Une, Cundinamarca, Colombia. Rev Inv Vet Peru 27: 751-757. doi: 10.15381/rivep.v27i4.12572

19. Hillyer GV, de Galanes MS, Buchón P, Bjorland J. 1996. Herd evaluation by enzyme-linked immunosorbent assay for the determination of Fasciola hepatica infection in sheep and cattle from the Altiplano of Bolivia. Vet Parasitol 61: 211-220. doi: 10.1016/03044017(95)00831-4

20. Howell A, Baylis $M$, Smith $R$, Pinchbeck G, Williams D. 2015. Epidemiology and impact of Fasciola hepatica exposure in high-yielding dairy herds. Prev Vet Med 121: 41-48. doi: 10.1016/j.prevetmed.2015.05.013

21. ITIS. 2019. Sistema Integrado de Información Taxonómica. Disponible en: http://www.itis.gov

22. Iturbe Espinoza P, Muñiz Pareja F. 2011. Desarrollo de huevos de Fasciola hepatica a partir de huevos aislados de la vesícula biliar de ovinos y vacunos, expuestos a luz y oscuridad. Neotrop Helminthol 5: 89-93.

23. Jara Campos C, Escalante Añorga $\mathrm{H}$, Cassana W, Davelois Atac K, Benites Murrieta A. 2018. Prevalencia de fascioliasis en ovinos y bovinos en la provincia de Pataz, La Libertad, Perú, mediante examen coproparasitológico y Western Blot. Rev Inv Vet Perú 29: 1421-1429. doi: 10.15381/rivep.v29i4.15198

24. Kajugu PE, Hanna REB, Edgar HW, McMahon C, Cooper M, Gordon A, Barley JP, et al. 2015. Fasciola hepatica: specificity of a coproantigen ELISA test for diagnosis of fasciolosis in faecal samples from cattle and sheep concurrently infected with gastrointestinal nematodes, coccidians and/or rumen flukes (paramphistomes), under field conditions. Vet Parasitol 212: 181187. doi: 10.1016/J.VETPAR.2015.07.018

25. Kooshan M, Hashemi T, Naghibi A. 2010. Use of somatic and excretorysecretory antigens of Fasciola hepatica in diagnosis of sheep by ELISA. Am Eurasian J Agric Environ Sci. 7: 170-175.

26. López-Villacís IC, Artieda-Rojas JR, Mera-Andrade RI, Muñoz-Espinoza MS, Rivera-Guerra V E, CuadradoGuevara AC, Zurita-Vásquez JH, et al. 2017. Fasciola hepatica: aspectos relevantes en la salud animal. J Selva Anim Sci 4: 137-146.

27. Marcilla A, Bargues MD, Mas-Coma S. 2002. A PCR-RFLP assay for the distinction between Fasciola hepatica and Fasciola gigantica. Mol Cell Probes 16: 327-333. doi: 10.1006/mcpr.2002.-0429 
28. Martínez-Valladares M, Robles-Pérez D, Martínez-Pérez JM, CorderoPérez $C$, Famularo $M$ del $R$, Fernández-Pato N, González-Lanza $C$, et al. 2013. Prevalence of gastrointestinal nematodes and Fasciola hepatica in sheep in the northwest of Spain: relation to climatic conditions and/ or man-made environmental modifications. Parasite Vector 6: 282. doi: 10.1186/1756-3305-6-282

29. Mas-Coma S, Bargues MD, Valero $M A .2005$. Fascioliasis and other plantborne trematode zoonoses. Int J Parasitol 35: 1255-1278. doi: 10.1016/J.IJPARA.2005.07 .010

30. Mas Coma S, Valero MA, Bargues MD. 2009. Fasciola, Lymnaeids and human fascioliasis, with a global overview on disease transmission, epidemiology, evolutionary genetics, molecular epidemiology and control. Adv Parasit 69: 41-146. doi: 10.1016/S0065308X(09)69002-3

31. Mehmood K, Zhang H, Sabir AJ, Abbas RZ, Ijaz M, Durrani $A Z$, Saleem MH, et al. 2017. A review on epidemiology, global prevalence and economical losses of fasciolosis in ruminants. Microb Pathog 109: 253-262. doi: 10.1016/j.micpath.2017.06.006

32. Ortega CM, Vásquez LR, Vargas $R$, Vergara D, Victoria I CL. 2017. Descripción epidemiológica de Fasciola hepatica en bovinos en el beneficiadero de Popayán, Cauca, Colombia. Rev Med Hond 85: 1-114.

33. Palacio Collado D, Bertot Valdés JA, Beltrao Molento M, Vázquez, Gil A, Izquierdo Pérez N, Arenal Cruz A, Arteaga Campbell A. 2017. Comportamiento estacional de Fasciola hepatica en bovinos sacrificados en el matadero Chacuba, Camagüey, Cuba. Rev Prod Anim 29: 30-35.

34. Pereira Silva AE, Freitas $C$ da C, Vieira Dutra L, Beltrão Molento M. 2016. Assessing the risk of bovine fasciolosis using linear regression analysis for the state of Rio Grande do Sul, Brazil. Vet
Parasitol 217: 7-13. doi: 10.1016/j.vetpar.2015.12.021

35. Pulido-Medellín MO, García-Corredor D, Díaz-Anaya A, Andrade-Becerra R. 2014. Pesquisa de parásitos gastrointestinales en pequeñas explotaciones ovinas. Rev Salud Anim 36: 65-69.

36. Quevedo LS, Bruhn FRP, Teixeira JLR, Alberti TS, Scheid HV, Raffi MB, Sallis E, et al. 2018. Epidemiological and clinical-pathological aspects of fasciolosis in livers of cattle slaughtered in southern Brazil. Pes Vet Bras 38: 17611766. doi: 10.1590/1678-5150-pvb-5880

37. Recalde-Reyes DP, Padilla Sanabria L, Isabel M, Giraldo Giraldo MI, Toro Segovia JL, González MM, Castaño Osorio JC. 2014. Prevalencia de Fasciola hepatica, en humanos y bovinos en el departamento del Quindío-Colombia. Infectio 18: 153 157. doi: 10.1016/ j.infect.2014.09.001

38. Rojo-Vázquez F, Meana A, Valcárcel F, Martínez-Valladares M. 2012. Update on trematode infections in sheep. Vet Parasitol 189: 15-38. doi: 10.1016/ j.vetpar.2012.03.029

39. Sargison N. 2012. Diagnosis of triclabendazole resistance in Fasciola hepatica. Vet Rec 171: 151.152. doi: 10.1136/vr.e5357

40. Sierra Balcárcel R, Martínez Vega R, Gutiérrez-Marín R, Dolores C, Uribe N. 2017. Estandarización de ELISA para el diagnóstico de fasciolosis bovina, ovina y humana. Salud UIS 49: 549556. doi: 10.18273/revsal.v49n4-2017004

41. Soca-Pérez M, Giupponi-Cardoso P, López-Vigoa O, Sanavria A, SánchezSantana T, Labrada-Vázquez A. 2016. Prevalencia de Fasciola hepatica en vacas en pastoreo durante el periodo poco lluvioso. Pastos y Forrajes 39: 281-285.

42. Uribe Delgado N, Sierra Balcárcel RF, Espinosa Gonzáles C. 2012. Comparación de las técnicas Kato-Katz, TSET y TSR en el diagnóstico de infección por Fasciola hepatica en humanos Salud UIS 44 7-12. 
43. Uruburu Gómez M, Bedoya Blandon J, Velásquez Trujillo L. 2013. ELISA indirecta para el diagnóstico de fasciolosis bovina en leche. Rev CES Med Vet Zoot 8: 93-100. doi: 10.21615/2850

44. Valderrama Pomé AA. 2016. Prevalencia de fascioliasis en animales poligástricos de Perú, 1985-2015. Rev Med Vet 32: 121-129. doi: 10.19052/ mv.3861

45. Valencia MN, Pariona DA, Huamán A M, Miranda M F, Quintanilla $C S$, Gonzáles A A. 2005. Seroprevalencia de fasciolosis en escolares y ganado vacuno en la Provincia de Huancavelica, Perú. Rev Peru Med Exp Salud Publica 22: 96-102.

46. Zaraei M, Arefkhah N, Moshfe A, Ghorbani F, Mikaeili F, Sarkari B. 2019. Prevalence of bovine fascioliasis in a new-emerging focus of human fascioliasis in Boyer Ahmad district, southwest of Iran. Comp Immunol Microb 66: 101350. doi: 10.1016/ j.cimid.2019.101350

47. Uribe Delgado N, Sierra Balcárcel RF, Espinosa Gonzáles C. 2012. Comparación de las técnicas Kato-Katz, TSET y TSR en el diagnóstico de infección por Fasciola hepatica en humanos. Rev Univ Ind Santander. 44(3), 7-12.

48. Uruburu Gómez M, Bedoya Blandon J, Velásquez Trujillo L. 2013. ELISA indirecta para el diagnóstico de fasciolosis bovina en leche. Rev CES Med Vet Zoot. 8(2): 93-100. Doi: $10.21615 / 2850$

49. Valderrama Pomé AA. 2016. Prevalencia de fascioliasis en animales poligástricos de Perú, 1985-2015. Rev Med Vet, (32): 121. Doi: 10.19052/mv.3861

50. Valencia $M N$, Pariona $D A$, Huamán A M, Miranda M F, Quintanilla C S, Gonzáles $A$ A. 2005. Seroprevalencia de fasciolosis en escolares y ganado vacuno en la Provincia de Huancavelica, Perú. Rev Peru Med Exp Salud Publica. 22(2): 96-102.

51. Zaraei M, Arefkhah N, Moshfe A, Ghorbani F, Mikaeili F, Sarkari B. 2019. Prevalence of bovine fascioliasis in a new-emerging focus of human fascioliasis in BoyerAhmad district, southwest of Iran. Comparative Immunol Microbiol Infect Dis, 66: 101350. doi: 10.1016/J.CIMID.2019.101350. 